\title{
Técnica para quantificação e qualificação de material coletado em filtros de proteção cerebral
}

\author{
Technique for quantifying and qualifying debris captured in embolic \\ protection filters
}

Gabriel Santos Novaes, Álvaro Razuk Filho, Geanete Pozzan, Andrea Reis, Alexandre Fioranelli,
Valter Castelli Jr., Walter Khegan Karakhanian, Roberto Augusto Caffaro*

\section{Resumo}

Contexto: A qualidade e a quantidade de partículas coletadas em filtros de proteção cerebral (FPC) durante angioplastia transluminal percutânea com stent (ATPS) podem esclarecer a importância desses dispositivos no tratamento de estenoses carotídeas.

Objetivos: Analisar o conteúdo retido por FPC em pacientes submetidos a ATPS de artéria carótida interna com nova técnica de análise qualiquantitativa.

Métodos: O material coletado em 10 FPC durante ATPS da bifurcação da carótida em pacientes com alto risco cirúrgico foi submetido a análise microscópica qualiquantitativa. Fotografias digitais das lâminas com material corado com hematoxilina e eosina foram analisadas com o programa Axio Vision LE Release 4.1, que calculou a área das partículas em micrômetros/metro quadrado $\left(\mu \mathrm{m}^{2}\right)$.

Resultados: O exame histopatológico evidenciou material em $100 \%$ dos filtros consistindo predominantemente de restos hemáticos, cristais de colesterol e cálcio. A área média de fragmentos coletados foi expressiva $\left(1.570 .310 \mu \mathrm{m}^{2}\right)$, e houve ampla variância desses valores.

Conclusões: Os FPC coletam quantidade importante de fragmentos de placas de ateroma, e a grande variância nas quantidades de material coletado pode estar associada com a gravidade da lesão, motivo pelo qual se tornam relevantes estudos que utilizem técnica padronizada para a quantificação desses fragmentos e para a compreensão de seu real significado clínico.

Palavras-chave: Artéria carótida, estenose carotídea, angioplastia transluminal percutânea com stent, filtros de proteção cerebral.

\section{Introdução}

A endarterectomia é o tratamento de eleição para a estenose da artéria carótida em pacientes selecionados ${ }^{1-5}$. Todavia, a angioplastia transluminal percutânea com implante de stent (ATPS) vem sendo cada vez mais aceita como alternativa para a cirurgia aberta em casos de risco cirúrgico elevado e doença cardiovascular obstrutiva ${ }^{6}$.

\begin{abstract}
Background: Quality and quantity of the content retained in embolic protection filters (EPFs) used in percutaneous transluminal angioplasty and stenting may possibly indicate the importance of EPFs in the management of carotid stenosis.
\end{abstract}

Objectives: To analyze the content retained by EPFs in patients undergoing percutaneous transluminal angioplasty and stenting of the internal carotid artery using a new technique for qualitative and quantitative analysis.

Methods: Material captured in 10 EPFs during percutaneous transluminal angioplasty and stenting in high-surgical-risk patients was examined to determine a qualitative and quantitative microscopic analysis. Digital photographs of the hematoxylin-eosin stained slides were analyzed using the Axio Vision LE Release 4.1 software in order to calculate the particles area in micra/square meter $\left(\mu \mathrm{m}^{2}\right)$.

Results: Histopathological examination identified particulate debris in $100 \%$ of the filters including predominantly blood residues, cholesterol crystals, and calcium Quantity of captured fragments was significant (mean of $1,570,310 \mu \mathrm{m}^{2}$ ) with a wide range of these values.

Conclusions: Significant quantity of fragments of atheromatous plaques is retained by EPFs and the wide range in the quantity of the retained debris can be associated with the lesion severity; therefore new studies using standardized technique for quantifying these fragments and for better understanding their real clinical meaning are necessary.

Keywords: Carotid artery, carotid stenosis, percutaneous transluminal angioplasty and stenting, embolic protection filters.

O sucesso da ATPS já foi muito questionado devido às altas taxas de eventos neurológicos relacionados com embolização distal decorrente da liberação de partículas durante a colocação do stent ${ }^{7}$. Embora nem todas as partículas liberadas sejam clinicamente importantes, evidências sugerem que o tamanho dessas partículas parece constituir o fator mais importante nesse fenômeno ${ }^{8-11}$.

\footnotetext{
* Disciplina de Cirurgia Vascular, Departamento de Cirurgia, Faculdade de Ciências Médicas da Santa Casa de São Paulo (FCMSCSP), São Paulo, SP.

Não foram declarados conflitos de interesse associados à publicação deste artigo.

Artigo submetido em 18.02.09, aceito em 04.11.09.

J Vasc Bras. 2009;8(4):307-312.

Copyright $\odot 2009$ by Sociedade Brasileira de Angiologia e de Cirurgia Vascular
} 
Bosiers et al. ${ }^{12}$ evidenciaram eventos tardios dependentes do tipo de stent utilizado (malha aberta ou fechada); por outro lado, já ficou demonstrado que a liberação dessas partículas é maior na dilatação após a colocação do stent ${ }^{13}$, o que levou ao desenvolvimento de sistemas de proteção que impedem que essas partículas ingressem na circulação cerebral $^{14-16}$.

Dentre esses dispositivos, os filtros de proteção cerebral (FPC) desenvolvidos para captura de partículas macroscópicas são os mais utilizados atualmente, e diversos estudos têm revelado taxas mais baixas de eventos neurológicos quando eles são utilizados na ATPS ${ }^{17-19}$.

O objetivo do presente estudo é a apresentação de uma técnica reprodutível para análise qualitativa e quantitativa dos fragmentos de placas de ateroma da bifurcação carotídea que são coletados pelos FPC.

\section{Método}

São apresentados neste estudo 10 procedimentos prospectivos de ATPS com FPC em artéria carótida. Esses procedimentos foram realizados na disciplina de Cirurgia Vascular da Faculdade de Ciências Médicas da Santa Casa de São Paulo (FCMSCSP), atendendo-se rigorosamente aos critérios para a indicação desse tratamento, ou seja, risco cirúrgico elevado, pescoço hostil e bifurcação carotídea alta. Foram incluídos apenas pacientes com estenose carotídea de origem aterosclerótica.

O grupo de pacientes submetidos aos procedimentos incluiu cinco homens e cinco mulheres com média de idade de $61,4 \pm 8,7$ anos (42 a 73 anos), dos quais cinco eram sintomáticos.

Antes da intervenção, os pacientes foram avaliados por angiografia cerebral diagnóstica por subtração digital (aparelho Integris V3000, Phillips ${ }^{\circledR}$ ) envolvendo o arco aórtico, todas as bifurcações das artérias carótidas e as artérias intracranianas, para a classificação das lesões com estenose da artéria carótida ${ }^{20}$. As lesões tratadas foram categorizadas nas classes angiográficas A (dois casos), B (seis casos) e C (dois casos) propostas por Wholey et al. ${ }^{20}$.

Com a devida aprovação do Comitê de Ética da FCMSCSP, o material coletado pelos FPC usados nas angioplastias foi analisado para os efeitos deste estudo.

ATPS e FPC: Os procedimentos foram realizados na Sala de Angiografia, Departamento de Radiologia da
FCMSCSP, com o aparelho Integris V 3000 (Phillips ${ }^{\circledR}$ ) e sob anestesia geral, por professores assistentes da disciplina de Cirurgia Vascular. Antes do procedimento, era ministrada dose de $100 \mathrm{mg}$ de ácido acetilsalicílico (AAS).

Após punção da artéria femoral utilizada como acesso arterial, os pacientes recebiam heparina sistêmica na dose de $100 \mathrm{U} / \mathrm{kg}$. Foi utilizado cateter guia 7F ou 8F Guider (Boston Scientific ${ }^{\mathbb{B}}$ ) ou bainha longa Shuttle Select (Cook Medical $^{\mathbb{R}}$ ), que, durante todo o procedimento, permanecia com irrigação contínua de solução fisiológica com heparina.

O FPC utilizado foi o FilterWire EZ (Embolic Protection System, Boston Scientific ${ }^{\circledR}$ ), composto de extremidade com mola espiral radiopaca, seguida de ponta cônica afilada, filtro de poliuretano com poros de 110 com alça de nitinol radiopaca, braço de suspensão do filtro e fio guia coberto com politetrafluoroetileno (PTFE) com diâmetro de 0,014 ". A extremidade cônica coberta com silicone em tamanho único (3.2F) é introduzida com segurança em vasos com diâmetros entre 3,5-5,5 $\mathrm{mm}$ e permite acesso fácil às lesões. A microporosidade do filtro permite fluxo sanguíneo contínuo ao mesmo tempo em que possibilita a captura eficaz de partículas embólicas. A alça suspensa de nitinol promove posicionamento de até $360^{\circ} \mathrm{em}$ vasos com anatomia tanto regular quanto tortuosa.

Não foi realizada pré-dilatação em nenhum paciente. Implantou-se stent autoexpansivo e de malha fechada Carotid Wallstent ${ }^{\circledR}$ (Boston Scientific $^{\mathbb{Q}}$ ) com perfil de 5 French, confeccionado com liga metálica denominada Elgiloy ${ }^{\circledR}$, composta de níquel (20\%), cobalto (40\%), cromo (20\%), molibdênio ( $7 \%$ ), magnésio (2\%), ferro, carbono e berílio, com densidade de $8,30 \mathrm{~g} / \mathrm{cm}^{3}$. A pós-dilatação foi realizada com o cateter balão Gazelle ou Ultrasoft (Boston Scientific $^{\circledR}$ ), com medidas de $5,0 \mathrm{~mm} \times 20 \mathrm{~mm}, 5,5 \mathrm{~mm} \times 20 \mathrm{~mm}$ ou $6,0 \mathrm{~mm} \times 20 \mathrm{~mm}$.

Análise do material coletado pelos filtros de proteção cerebral: Após a realização da ATPS com FPC, esses dispositivos eram retirados e abertos para a avaliação macroscópica do seu interior. Coágulos aderidos na malha do filtro não eram considerados partículas macroscópicas. Em seguida, eram fechados, armazenados em solução de formol a $10 \%$ e encaminhados ao Departamento de Patologia da FCMSCSP, onde eram abertos novamente e lavados com solução de formol. As partículas eram citocentrifuga- 
das em Centrífuga Hermle Z300, e o material resultante era colocado em lâminas e corado com hematoxilina e eosina.

As lâminas foram avaliadas em microscópio Zeiss Axioskop 40, com aumento de 10 vezes, e o aglomerado de partículas localizado na lâmina era fotografado com máquina fotográfica digital Sony, modelo DSC-S85, com quatro MP na resolução 1280 x 960.

As fotos de cada lâmina foram analisadas pelo programa Axio Vision LE Release 4.1, desenvolvido para possibilitar a caracterização morfológica do material analisado e para calcular a área de partículas em micrômetros por metro quadrado $\left(\mu \mathrm{m}^{2}\right)$. As células sanguíneas porventura encontradas nas amostras não eram consideradas para medição.

\section{Resultados}

Ao exame, após a abertura dos filtros, foram identificadas partículas macroscópicas em dois (20,0\%) deles (Figura 1).

Após a coloração por hematoxilina e eosina foi possível realizar, através da observação por microscopia óptica, uma avaliação qualitativa da composição das partículas aprisionadas pelos filtros, que se mostraram constituídas por restos hemáticos, cálcio e cristais de colesterol em todos os filtros examinados (Figura 2). A presença do cálcio e dos cristais de colesterol deve-se à fragmentação da placa do ateroma.

$\mathrm{Na}$ análise quantitativa, o cálculo da área das partículas capturadas pelos filtros realizado com o programa Axio Vision LE Release 4.1 (Figura 3) resultou em valores que oscilaram entre $2.480,33$ e $13.568 .860,21 \mu \mathrm{m}^{2}$, com a mé-

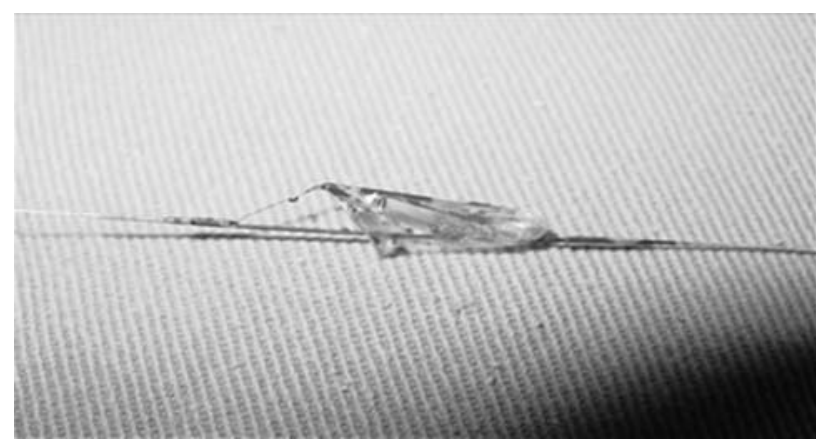

Figura 1 - Aspecto macroscópico de partículas coletadas por filtro de proteção cerebral após angioplastia transluminal percutânea com stent

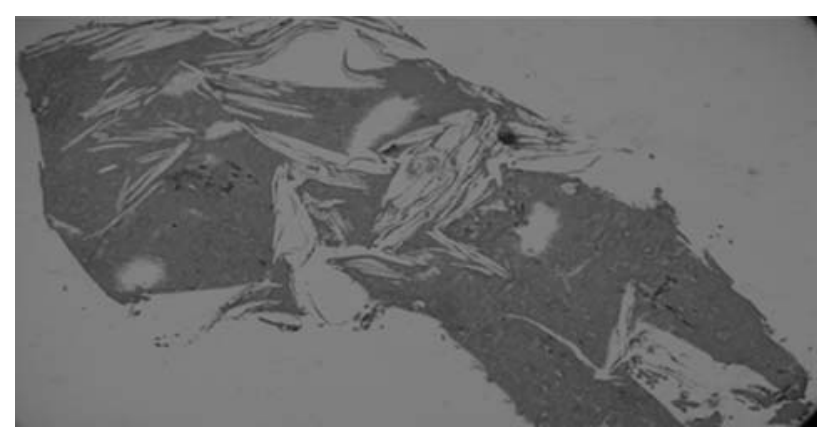

Figura 2 - Imagem de microscopia óptica com aumento de 10 vezes e coloração com hematoxilina e eosina em que se observam sinais de colesterol, restos hemáticos e cálcio

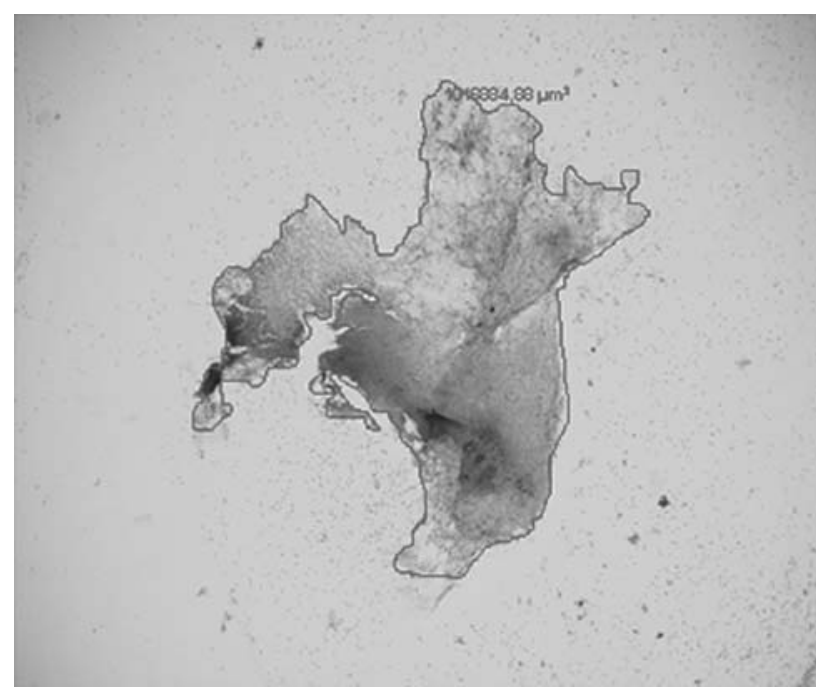

Figura 3 - Exemplo de área calculada pelo programa Axio Vision LE Release 4.1 do aglomerado de partículas coletadas por filtros de proteção cerebral

dia de $1.570 .310 \mu \mathrm{m}^{2}$ e variância significativamente ampla $\left(\mathrm{F}=0,003 ; \mathrm{DP}=4.223 .240 \mu \mathrm{m}^{2}\right)$.

\section{Discussão}

O material capturado pelos FPC nesta série consistiu, totalmente, de partículas de origem ateromatosa liberadas pela ruptura das placas de ateroma durante a angioplastia, confirmando achados histológicos de outros estudos ${ }^{17,21,22}$. DeRubertis et al. ${ }^{21}$ observaram quantidade irrisória de cálcio no material coletado e relataram ativação plaquetária importante em material coletado de pacientes sintomáticos. A melhor compreensão da composição da placa de ateroma e das partículas geradas durante a ATPS, que é possibilitada pela análise de material coletado pelos FPC, poderá conduzir, em última instância, ao desenvolvimento 
de estratégias focadas na redução de eventos neurológicos durante e após o tratamento da estenose carotídea.

A captura de material macroscópico (20\%) foi bastante inferior aos $60,3^{6}$ a $69 \%{ }^{18}$ relatados na literatura. Sprouse et al. ${ }^{6}$ utilizaram cinco filtros diferentes e, sem avaliar fatores microscópicos do material coletado, concluíram que os FPC são dispensáveis em angioplastias, já que não preveniram macroembolização em $40 \%$ dos casos. Nossa coleta de material macroscópico por FPC foi apenas ligeiramente superior aos $19 \%$ relatados por DeRubertis et $\mathrm{al}^{21}$, ressaltando-se que utilizamos o mesmo filtro em todos os casos.

DeRubertis et al. ${ }^{21}$ coletaram material microscópico em $77 \%$ dos filtros estudados; utilizaram e compararam diferentes filtros e analisaram a linha de tempo de uso desses dispositivos, concluindo que a coleta efetiva de partículas microscópicas depende, diretamente, do tipo de filtro utilizado e da curva de aprendizagem, o que já havia sido observado por Cremonesi et al. ${ }^{17}$.

Nossos resultados indicaram que 100\% dos FPC capturaram debris com área média de $1.570 .310 \mu \mathrm{m}^{2}$ (de 2.480,33 a 13.568.860,21 $\mu \mathrm{m}^{2}$ ), e essa ampla variância parece estar correlacionada com a gravidade das lesões, uma vez que debris com áreas maiores foram mais frequentes entre os pacientes com lesões angiograficamente mais graves (Classe $\mathrm{C}$ de Wholey ${ }^{20}$ ).

A porcentagem de filtros que coletaram material microscópico para análise (100\%) foi superior à relatada por outros autores, que variaram de $77,0^{21}$ a $83,7 \%{ }^{17}$.

Não há métodos idealmente padronizados para determinação da quantidade ou do tamanho de debris coletados em FPC nos diferentes estudos, mas a grande variância dos valores encontrados em nosso estudo ratifica outros acha$\operatorname{dos}^{17,19,21,23}$, suscitando o interesse de entendê-la melhor em relação às características clínicas e angiográficas dos pacientes com estenoses carotídeas.

Ressalte-se, contudo, que, nos demais estudos que se ocuparam em proceder à análise do material coletado por FPC, as amostras foram observadas por microscopia eletrônica de varredura ${ }^{17-19,21}$, que é uma técnica mais onerosa e menos acessível. Considerando que os resultados deste estudo não se distanciam absolutamente das observações feitas por outros pesquisadores, o uso da microscopia óptica para a análise de material coletado por FPC mostra-se tão eficaz quanto o uso da microscopia eletrônica de varredura, com a vantagem de ser menos onerosa e mais acessível, permitindo reprodutibilidade da técnica mais fácil e mais rápida, o que, por sua vez, pode inclusive agilizar o entendimento das eventuais correlações entre esse material coletado por FPC e a prevenção de quadros neurológicos após ATPS.

Parece que fatores clínicos representados por comorbidades associadas podem ser preditivos de maior ou menor tamanho de partículas coletadas por FPC, embora não sejam preditivos da ausência dessas partículas em FPC utilizados em ATPS $^{6}$. A presença, quantidade ou tamanho dessas partículas tampouco têm valor preditivo para novas lesões isquêmicas posteriores à ATPS com FPC ${ }^{22}$.

Há uma teoria segundo a qual as características da lesão com estenose podem determinar sua propensão para originar êmbolos e, portanto, o seu potencial para ocasionar um acidente vascular encefálico. Neste sentido, a quantidade de partículas capturadas estaria associada com o grau da lesão com estenose ${ }^{24}$. Há, contudo, poucos estudos voltados para essa associação, embora já tenha sido observado que artérias comprometidas por lesões mais graves podem estar mais propensas às lesões histológicas causadas por $\mathrm{FPC}^{18,24}$.

Nossos achados permitem abonar as recomendações segundo as quais os FPC devem ser usados com maior cuidado em lesões mais avançadas, especialmente naquelas excessivamente anguladas ou nas que a passagem do dispositivo requer força excessiva ${ }^{19}$. Lesões $>2 \mathrm{~cm}$ também estão associadas com probabilidade três vezes maior de complicações neurológicas ${ }^{20}$, o que também adverte em relação a cuidados especiais e curva de aprendizagem maior na utilização de ATPS com FPC. A avaliação angiográfica para determinação do grau de lesão com estenose antes da revascularização da artéria carotídea é conduta indispensável.

Já foi fortemente evidenciado que o tipo de malha do stent utilizado explica a maior taxa de complicações tardias em população sintomática, o que não ocorre em pacientes assintomáticos ${ }^{12}$. Estudos mais focados no uso de $\mathrm{FPC}^{7,21}$ não conseguiram reproduzir essas diferenças, relatando resultados homogêneos para pacientes com e sem sintomatologia neurológica, tanto no que se refere à evolução peri e pós-intervenção terapêutica quanto às características do material coletado. 
Acreditamos que os estudos do material coletado por FPC usados com os cuidados devidos e com técnicas e materiais adequados durante ATPS ainda trarão informações preciosas para a compreensão dos mecanismos que levam à embolização e a eventos neurológicos nesses procedimentos. A técnica utilizada neste estudo evidenciou a grande quantidade de material coletado pelos filtros de proteção, que, assim, deixou de ingressar na circulação intracraniana, confirmando o potencial desse sistema de proteção para reduzir complicações neurológicas durante o tratamento da estenose da artéria carótida.

\section{Conclusão}

O presente estudo permite concluir que o método utilizado para avaliação qualitativa e quantitativa das partículas capturadas pelos FPC é efetivo, menos oneroso e mais acessível e rápido, e permitirá análise e correlação com dados clínicos e anatômicos.

\section{Referências}

1. Barnett HJ, Taylor DW, Eliasziw M, et al. Benefit of carotid endarterectomy in patients with symptomatic moderate or severe stenosis. North American Symptomatic Carotid Endarterectomy Trial Collaborators. N Engl J Med. 1998;339:1415-25.

2. Beneficial effect of carotid endarterectomy in symptomatic patients with high grade carotid stenosis. North American Symptomatic Carotid Endarterectomy Trial Collaboration. N Engl J Med. 1991;325:445-53.

3. MRC European Carotid Surgery Trial: Interim results for symptomatic patients with severe (70-99\%) or with mild (0-29\%) carotid stenosis. The European Carotid Surgery Trialists' Collaborative Group. Lancet. 1991;337:1235-43.

4. Endarterectomy for moderate symptomatic carotid stenosis: interim results from de MRC European Carotid Surgery Trial. Lancet. 1996;347:1591-3.

5. Randomized trial of endarterectomy for recently symptomatic carotid stenosis: Final results of the MRC European Carotid Surgery Trial (ECST). Lancet. 1998;351:1379-87.

6. Sprouse LR 2nd, Peeters P, Bosiers M. The capture of visible debris by distal cerebral protection filters during carotid artery stenting: Is it predictable? J Vasc Surg. 2005;41:950-5.

7. Yadav IS, Wholey MH, Kuntz RE, et al. Protected carotidartery stenting versus endarterectomy in high risk patients. $\mathrm{N}$ Engl J Med. 2004;351:1493-501.

8. Crawley F, Clifton A, Buckenham T, Loosemore T, Taylor RS, Brown MM. Comparison of hemodynamic cerebral ischemia and microembolic signals detected during carotid endarterectomy and carotid angioplasty. Stroke. 1997;28:2460-4.

9. Manninen HI, Rasanen HT, Vanninen RL, Vainio P, Hippeläinen M, Kosma VM. Stent placement versus percutaneous transluminal angioplasty of human carotid arteries in cadaver in situ: distal embolization and findings at intravascular. Radiology. 1999;212:483-92.

10. Parodi JC, Ferreira LM, Sicard G, La Mura R, Fernandez S. Cerebral protection during carotid stenting using flow reversal. J Vasc Surg. 2005;41:416-22.

11. Rapp JH, Wakil L, Sawhney RS, et al. Subclinical embolization after carotid artery stenting: new lesions of diffusion-weighted magnetic resonance imaging occur postprocedure. J Vasc Surg. 2007;45:867-72.

12. Bosiers M, de Donato G, Deloose K, et al. Does free cell area influence the outcome in carotid artery stenting? Eur J Vasc Edovasc Surg. 2007;33:135-41.

13. Burton KR, Lindsay TE. Assessment of short-term outcomes for protected carotid angioplasty with stents using recent evidence. J Vasc Surg. 2005;42:1094-100.

14. Mellière D. [Carotid surgery: assessment and current problems]. J Mal Vasc. 1993;18:176-85.

15. Ohki T, Marin NL, Lyon RT, et al. Ex vivo human carotid artery bifurcation stenting: Correlation of lesion characteristics with embolic potential. J Vasc Surg. 1998;27:463-71.

16. Theron J, Courtheoux P, Alachkar F, Bouvard G, Maiza D. New triple coaxial catheter system for carotid angioplasty with cerebral protection. Am J Neuroradiol. 1990;11:869-74.

17. Angelini A, Reimers B, Della Barbera M, et al. Cerebral protection during carotid artery stenting: collection and histopathologic analysis of embolized debris. Stroke. 2002;33:456-61.

18. Cremonesi A, Manetti R, Setacci F, Setacci C, Castriota F. Protected carotid stent: Clinical advantages and complications of embolic protection devices in 442 consecutive patients. Stroke. 2003;34:1936-41.

19. Reimers B, Corvaja N, Moshiri S, et al. Cerebral protection with filter devices during carotid artery stenting. Circulation. 2001;104:12-5.

20. Wholey MH, Wholey M, Mathias K, et al. Global experience in cervical carotid artery stent placement. Catheter Cardiovasc Intervent. 2000;50:160-7.

21. DeRubertis BG, Chaer RA, Gordon R, et al. Determining the quantity and character of carotid artery embolic debris by electron microscopy and energy dispersive spectroscopy. J Vasc Surg. 2007;45:716-25.

22. Maleux G, Demaerel P, Verbeken E. Cerebral ischemia after filter-protected carotid artery stenting is common and cannot be predicted by the presence of substantial amount of debris captured by the filter device. Am J Neuroradiol. 2006;27:1830-3.

23. Whitlow PL, Lylyk P, Londero H, et al. Carotid artery stenting protected with an emboli containment system. Stroke. 2002;33:1308-14.

24. Müller-Hülsbeck S, Stolzmann P, Liess C, et al. Vessel wall damage caused by cerebral protection devices: Ex vivo evaluation in porcine carotid arteries. Radiology. 2005;235:45460. 
Correspondência:

Gabriel Santos Novaes

Rua Cesário Motta Jr., 121, Vila Buarque

CEP 01221-020 - São Paulo, SP

Tel./Fax: (11) 2176.7273

E-mail: drgabrielnovaes@terra.com.br

\section{Contribuições dos autores}

Concepção e desenho do estudo: AR Filho, GSN

Análise e interpretação dos dados: VC Junior, GSN
Coleta de dados: AR, GP

Redação do artigo: GSN

Revisão crítica do texto: WKK

Aprovação final do artigo*: RAC, AR Filho, GSN

Análise estatística: AF

Responsabilidade geral pelo estudo: GSN

Informações sobre financiamento: N/A

*Todos os autores leram e aprovaram a versão final submetida ao J Vasc Bras. 